\title{
Protein Disulfide Isomerase and Host-Pathogen Interaction
}

\author{
Beatriz S. Stolf, ${ }^{1}$ loannis Smyrnias, ${ }^{2}$ Lucia R. Lopes, ${ }^{3}$ Alcione Vendramin, ${ }^{4}$ Hiro Goto, ${ }^{5}$ \\ Francisco R. M. Laurindo, ${ }^{6}$ Ajay M. Shah, ${ }^{2}$ and Celio X. C. Santos ${ }^{2}$ \\ ${ }^{1}$ Department of Parasitology, University of São Paulo, São Paulo, SP, Brazil \\ ${ }^{2}$ Cardiovascular Division, British Heart Foundation Centre, King's College London, \\ 125 Coldharbour Lane, London SE5 9NU, UK \\ ${ }^{3}$ Department of Pharmacology, Institute of Biomedical Sciences, University of \\ São Paulo, São Paulo, SP, Brazil \\ ${ }^{4}$ Department of Parasitology, School of Medicine of Jundiaí, São Paulo, SP, Brazil \\ ${ }^{5}$ School of Medicine and Tropical Medicine Institute, University of São Paulo, \\ São Paulo, SP, Brazil \\ ${ }^{6}$ Vascular Biology Laboratory, Heart Institute (InCor), São Paulo, Brazil
}

Received 3 June 2011; Accepted 7 September 2011

Academic Editor: Mauro Perretti

Reactive oxygen species (ROS) production by immunological cells is known to cause damage to pathogens. Increasing evidence accumulated in the last decade has shown, however, that ROS (and redox signals) functionally regulate different cellular pathways in the host-pathogen interaction. These especially affect (i) pathogen entry through protein redox switches and redox modification (i.e., intra- and interdisulfide and cysteine oxidation) and (ii) phagocytic ROS production via Nox family NADPH oxidase enzyme and the control of phagolysosome function with key implications for antigen processing. The protein disulfide isomerase (PDI) family of redox chaperones is closely involved in both processes and is also implicated in protein unfolding and trafficking across the endoplasmic reticulum (ER) and towards the cytosol, a thiol-based redox locus for antigen processing. Here, we summarise examples of the cellular association of host PDI with different pathogens and explore the possible roles of pathogen PDIs in infection. A better understanding of these complex regulatory steps will provide insightful information on the redox role and coevolutional biological process, and assist the development of more specific therapeutic strategies in pathogen-mediated infections.

KEYWORDS: Host, pathogen, redox, endoplasmic reticulum, parasites, PDI, Nox 


\section{INTRODUCTION}

Host cells have the ability to cope with the progression and severity of infection in response to different types of pathogen. On the other hand, numerous mechanisms have evolved that support the use of the host cell machinery to facilitate pathogen survival and multiplication. Such co-evolutionary processes are directly affected by different physicochemical factors within different cell compartments, both in the host and in pathogen. For instance, $\mathrm{pH}$ critically affects antigen stability of the influenza virus which modulates endosome acidity that attenuates its own infection [1]. ROS (and Reactive Nitrogen Species) production and the redox state of different cell compartments are also critically involved in cellular hostparasite interaction. Among the many redox sensitive proteins that are altered during the course of different infections, protein disulfide isomerase (PDI-) mediated redox switches have been associated with pathogen attachment-internalization, antigen processing in the ER/phagosome, and the regulation of ROS production by Nox family enzymes. Thus, PDI emerges as a ubiquitous redox protein that regulates different steps of diverse infection processes. Several pathogens also have their own PDI that act as an important virulence factor (Table 1). Other redox modifications directly mediated by ROS and especially via nitric oxide (NO) generated by inducible nitric oxide synthase (iNOS), which is abundant in phagocytic cells, have been reviewed elsewhere $[2,3]$ and are not considered in this article. Below, the main cellular redox aspects of host and pathogen PDI will be discussed.

\section{GENERAL ASPECTS OF PDI AND ITS ROLE IN HOST AND PATHOGEN}

The ancient PDI is a ubiquitous redox chaperone belonging to the thioredoxin oxireductase super family and can reduce (reaction 1), oxidize (reaction 2), and catalyse dithiol-disulfide exchange reactions (i.e., isomerase activities, reaction 3, Figure 1). Such broad range of activities overlaps with the chaperone role of PDI that overall performs a housekeeping function in helping to maintain proteins in a more stable conformation. There are around 20 PDI homologues, and the detailed structure and function of eukaryotic PDIs have been covered in recent excellent reviews [4, 5]. The classic mammalian PDI (55 kDa) has several domains ordered as a-b-b'-a'-c with 2 thioredoxin-like motifs (Trp-Cys-Gly-His-Cys) displayed in the a and $\mathrm{a}^{\prime}$ domain [4-6] (Figure 1). PDI is abundant in the ER $(\sim 0.5 \mathrm{mM})$ where the relatively oxidizing conditions at basal level (i.e., GSH/GSSG ratios 2-3:1) favours PDI isomerase/oxidase activity, which is primarily involved in client protein redox folding (reaction 2-3, Figure 1). The oxidizing equivalents for this process are driven mainly by the ER thiol-containing oxidase, Ero1 (endoplasmic reticulum oxidase1), which binds FAD and is in turn re-oxidized via electron transfer to oxygen, generating $\mathrm{H}_{2} \mathrm{O}_{2}$ in the process [7-10]. The $\mathrm{H}_{2} \mathrm{O}_{2}$ destiny is elusive, but it can oxidize ER-located peroxiredoxin IV (PrxIV) that is further reduced by PDI that is oxidized in the process [11]. This redox circuit is thought to increase total protein folding and thiol oxidation via Ero1 [11]. However, even in the absence of Ero1, protein folding still occurs, and it is suggested that other oxidases may compensate for redox demand in the ER in some circumstances $[12,13]$. Nevertheless, the PDI-Ero1-dependent oxidative activity is balanced to cytosolic glutathione levels suggesting a functional redox interplay between these compartments [12]. PDI reductase activity has been primarily associated to more reducing compartments (i.e., GSH/GSSG ratios $\sim 30-100: 1$ ), such as those in the vicinity of the plasma membrane $[6,10]$. PDI redox versatility is mainly governed by the low $\mathrm{pKa}$ of the proximal cysteine on the active $\mathrm{N}$-terminal a domain. Indeed, the lower pKa of 4.5 renders PDI a much better oxidase than thioredoxin, which has a pKa of 7.1 and is mainly a reductase in most neutral $\mathrm{pH}$ cell compartments. It should also be noted that PDI functions as a chaperone independently of its redox-active domains as especially required for its ATPase and $\mathrm{Ca}^{2+}$ activity, although PDI redox motifs still stabilize binding interaction $[4,5,10]$. In the ER, PDI is tightly associated with prolyl-4 hydroxylase (the rate-limiting enzyme for collagen biosynthesis), the Sec61 translocon, and the MHC class I complex (see later). It can be also found as a heterodimer with microsomal triglyceride transfer protein $[6,10]$. PDI is a soluble homodimer and does not have a transmembrane domain and, similarly to other ER chaperones, carries the KDEL C-terminal sequence which binds to respective receptors in the COP 
TABLE 1: Summary of the main PDI types and redox activity expressed in different groups of pathogens.

\begin{tabular}{lccc}
\hline Pathogen group & PDI (thioredoxin motif) & Redox activity & Reference \\
\hline Leishmania major & 5 isoforms (CGHC, CRYC) & Oxidase-isomerase & {$[16,23,24]$} \\
amazonensis & 5 isoforms (CGHC, CRYC) & Reductase-isomerase & {$[16,23,24]$} \\
infantum & 5 isoforms (CGHC) & ND & {$[23]$} \\
braziliensis & 3 isoforms (CGHC) & ND & {$[23]$} \\
\hline Trypanosoma cruzi & 5 isoforms (CXXC) & ND & {$[25,26]$} \\
\hline Plasmodium falciparum & 4 isoforms (2x CGHC) & Oxidase-reductase & {$[27,28]$} \\
\hline Toxoplasma gondii & $\mathrm{ND}$ & ND & {$[29]$} \\
\hline Entamoeba histolytica & 1 isorform, 38 kDa (2x CGHC) & Oxidase-reductase & {$[30]$} \\
\hline
\end{tabular}

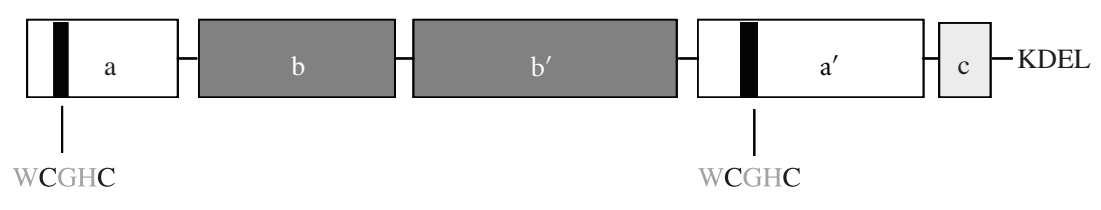

(1) PDI $(-\mathrm{SH},-\mathrm{SH})+\mathrm{Pt}(-\mathrm{S}-\mathrm{S}-) \stackrel{\text { Reduction }}{\longrightarrow} \mathrm{PDI}(-\mathrm{S}-\mathrm{S}-)+\mathrm{Pt}(-\mathrm{SH},-\mathrm{SH})$
(2) PDI $\left(-\mathrm{S}_{-} \mathrm{S}-\right)+\mathrm{Pt}(-\mathrm{SH},-\mathrm{SH}) \stackrel{\text { Oxidation }}{\longrightarrow} \mathrm{PDI}(-\mathrm{SH},-\mathrm{SH})+\mathrm{Pt}(-\mathrm{S}-\mathrm{S}-)$

(3) PDI (-S-S-) + Pt (-S-S-, SH, SH) $\stackrel{\text { Isomerization }}{\longrightarrow} \mathrm{PDI}\left(-\mathrm{S}_{-} \mathrm{S}-\right)+\mathrm{Pt}\left(-\mathrm{SH},-\mathrm{S}_{-}-\mathrm{S}_{-},-\mathrm{SH}\right)$

FIGURE 1: PDI and its main reactions: upper, general display of the five domains of mammalian PDI protein and showing the position of the active thioredoxin motif (WCGHC); lower, the main thiol redox reactions driven by PDI on Pt (protein/peptide) substrate containing thiols. Note that in reaction 3, the oxidation is driven by $\mathrm{H}_{2} \mathrm{O}_{2}$, especially in the oxidising ER compartment.

vesicles that circulate in the ER-Golgi vicinity, recycling proteins back to ER. PDI also undergoes intense intracellular trafficking and is found on the surface of diverse prokaryotic and eukaryotic cells [14-16]. Despite limited knowledge about this traffic, it is possible that PDI exits the ER through the translocon Sec61 pore and/or via secretory vesicles [17]. PDI is thought to attach to lipids, glycans, and integral membrane proteins via electrostatic interactions at the cell plasma membrane $[14,15]$, where its reductive activity mediates the infection of different pathogens (Figure 2, discussed later). PDI along with ERp57 have been found in the nuclei in association to DNA and affecting transcriptional activity of NF-kb, AP-1, and STAT3 [15]. These transcriptional regulators are key elements in many inflammatory processes, but their functional association to PDI and to different pathogen still elusive. In contrast to many other members of its family, such as thioredoxin itself and Erp57, PDI is not normally found in the cytosol, where it is likely cleaved by caspase-3 and -7 [18].

Protozoans and bacteria have their own PDIs (Table 1). The function of these PDIs in protein folding is poorly understood; however, there are intriguing data correlating PDI expression and the pathogenicity of several parasites, especially obligatory intracellular protozoans. Leishmania that leads to distinct types 


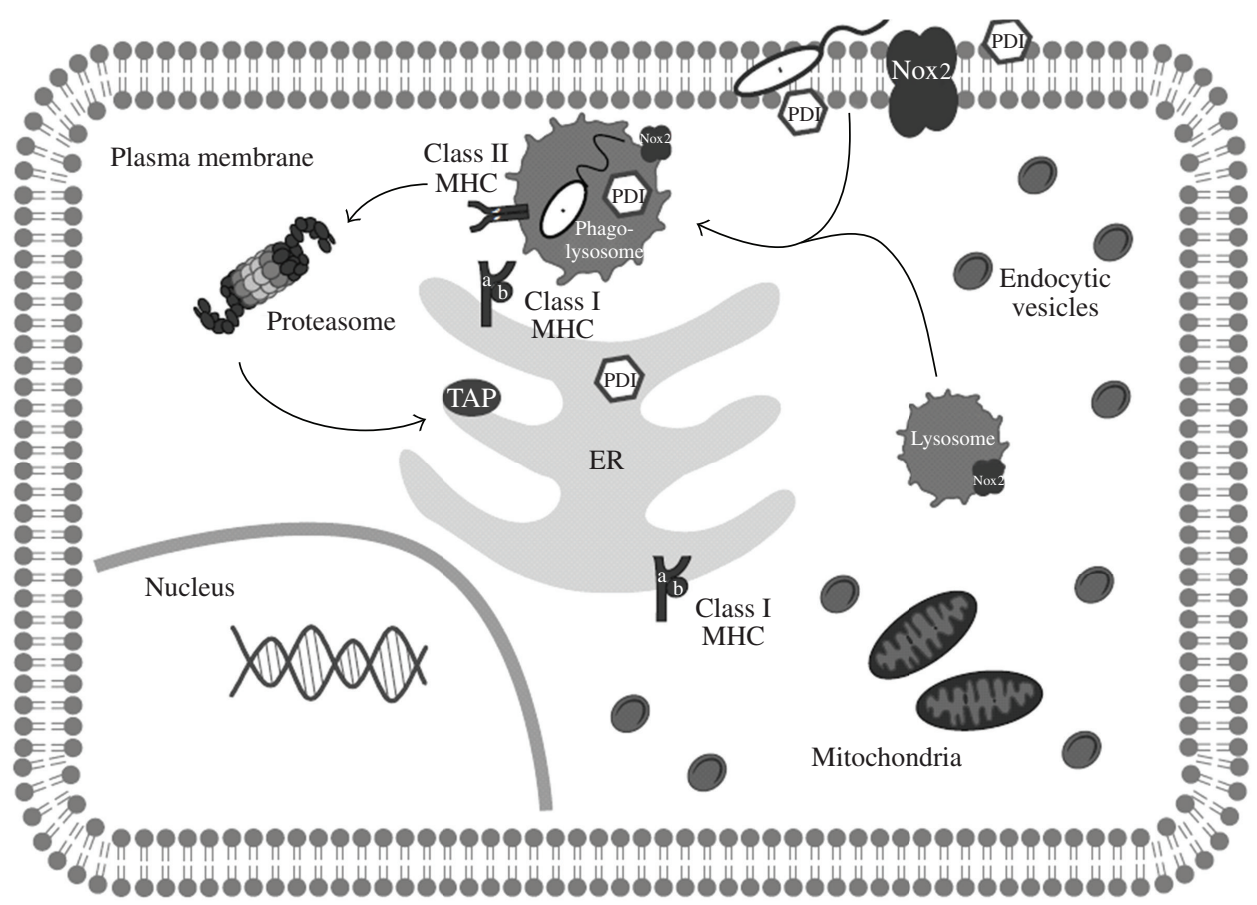

FIGURE 2: Main cellular routes involved in host mammalian PDI and pathogens.

of leishmaniases is a well-known example. The disease affects millions of individuals worldwide, and more recently this parasite emerged as an important opportunistic infection among patients with HIV [19]. There is a large variety of Leishmania species that results in different infection manifestation, generally classified as cutaneous (L. major, L. mexicana, L. amazonensis, L. braziliensis, etc.), diffuse cutaneous $(L$. amazonensis), mucocutaneous (L. braziliensis), and visceral (L. donovani, L. chagasi, and/or L. infantum) [20]. If $L$. chagasi is a subgroup of $L$. infantum brought to America by European colonist or other specie in its own still a matter of controversy (see discussion in [21]). The infection cycle in the vertebrate host and is initiated when Leishmania promastigote is injected into the skin by the insect vector. In the host, the promastigote is phagocytised especially by macrophages, and further it is converted into intracellular amastigote. Amastigote replicates inside the phagosome within the cell and is liberated after the cell lyses, subsequently infecting other cells resulting in the progression to disease [21,22]. L. amazonensis has at least four PDIs, and the use of specific PDI inhibitors substantially affected parasite growth [23]. In L. major, the increased levels of Leishmania PDI (LmPDI) expression and secretion at the parasite surface reflects optimal protein folding balanced to parasite multiplication. Importantly, this is correlated to high virulence of the parasite strains [16]. More recently, the use of LmPDI antigens to generate a vaccine for L. major partially protected BALB/c animals and accelerated the cure of different strains of mice [24]. Similarly to Leishmania species, other parasites of the trypanosomatid group such as Trypanosoma contain several genes predicted to encode for PDIs, that can execute $N$-glycosylation and protein folding in the ER [25, 26]. Although PDI was considered essential for T. brucei survival, PDI activity was not essential for the growth of trypanosomes in vitro [26]. PDI is also expressed in different species of Plasmodium protozoans (Table 1), the parasites that cause malaria $[27,28]$. P. falciparum expresses at least nine different PDIs and the PfPDI8 has great similarity to the prototype PDI and is expressed during all stages of parasite life cycle. This PDI facilitates the disulfide-dependent conformational folding of EBA-175 protein, an emerging candidate for the development of malaria vaccines [28]. This is intriguing given that malaria parasites express proteins with high content of cysteine, which are associated to parasite invasion and sequestration in the vertebrate host and transmission into mosquito host [28]. Finally, Toxoplasma gondii PDI was identified in host tears, suggesting an extracellular location and adhesion to host cells during the initial phase of infection [29]. 


\section{PDI AND HOST-PATHOGEN INTERACTION}

Antigen presentation occur through two distinct pathways. Antigen presenting cells (APCs; especially macrophages and dendritic cells; DCs) are long-lived cells that capture antigens and subsequently process and present them at the cell surface, where they are recognized by T-lymphocytes. This process provides a long-term adaptive immune response to fungi, bacteria, and parasite. After internalization by the APC, antigens pass through phagosome/lysosome vesicles, where they form complexes with MHC class II (Figure 2), which are recognized by helper CD4+ T lymphocytes (exogenous pathway). In contrast, self cell antigens and virus synthesized within cells (mostly non-APCs) are degraded by the proteasome in the cytosol and nucleus. In successive steps, the antigen is processed, folded, and incorporated into the MHC class I (Figure 2) and the complex exposed on the cell surface, and recognized by cytotoxic CD8+ T lymphocytes (endogenous pathway). These two pathways overlap and some antigens are presented by both MHC class I and II, in a process called cross-presentation. This has been described in DCs responding to viral infection, transplant rejection, and some autoimmune diseases and cancer. Moreover, a wide range of pathogens passing or living in the phagosome such as Mycobacterium tuberculosis, Salmonella typhimurium, Toxoplasma gondii, and especially Leishmania spp and Trypanosoma cruzi are all crosspresented in association to high levels of $\mathrm{CD} 8^{+} \mathrm{T}$ cells [31]. PDI as part of the ER protein folding machinery directly regulates antigen processing of the MHC class I complex [32-35]. Antigens that are degraded by peptidases and proteasome to shorter peptides in the cytosol and nucleus can be further transported to the ER through the TAP system, a transmembrane ER type of ATP-binding cassette (ABC) peptide transporter family [36]. ER-located PDI interacts with the peptide-loading complex (PCL) that efficiently promotes peptide assembly with MHC class I molecules and supporting the exit of the peptide-antigen complex from the ER [32-34]. Other PCL components include calreticulin, tapasin, ERp57 (another PDI family member), and the TAP transporter itself. Cells lacking PDI present much less peptide loading to MHC class I and the disulfide bridge between the peptide and MHC groove remains in a reduced redox state [32]. Normally, this interaction is affected by the redox exchange between PDI (predominantly oxidized) and ERp57 (predominantly reduced) [32], a condition in which PDI favours the release of peptide-MHC class I from the PCL and the antigen-MHCI complex is exited from ER [34]. In fact, PDI-bound peptide facilitates the disassembly of the tapasin-ERp57 complex while the PDI unbound to the complex is unable to interact with tapasin-ERp57, retaining MHC I molecules in the ER [34]. Overall, PDI redox activity modulates the stability of the antigen peptide-MHC class I complex and further determines the transport of the complex to the plasma membrane [32-35]. These redox effects may vary according to the type of antigen and some pathogens interfere with this pathway to escape antigen process and evading $\mathrm{CD}^{+}$ T-cells recognition. This is the case for US3 protein from human cytomegalovirus, which enhances PDI degradation via the proteasome [32]. PDI participation in immune response, however, goes beyond its role in the ER protein folding machinery and it acts at other cellular steps of host-pathogen interaction. PDI in the ER is also thought to play a role in parasite phagocytosis, and the PDI displayed on the cell surface can mediate the entry of some viral, bacterial, and protozoan. PDI is also implicated in protein unfolding and trafficking of some pathogenic antigens across the endoplasmic reticulum and towards the cytosol by the endoplasmic reticulum-associated degradation system (ERAD). This is the main pathway where proteins are retrotranslocated from the ER to cytosol and further degradated by the proteasome. Next, we discuss some examples of the cellular association between host PDI and different pathogens.

\subsection{PDI and the Phagocytic Process}

Phagocytosis is the main gate for large microbes to enter into APCs. After binding and attaching to the pathogen, these cells can internalize organisms and large particles even bigger then their own size, which are then phagocytosed in an active process that involves intense membrane remodelling [37]. Proteomics studies accumulated over the last decade revealed the presence of ER chaperones in the isolated phagosome, uncovering a process called ER-mediated phagocytosis [38-45]. ER chaperones were detected 
in phagosomes of macrophages exposed to different particulate material and pathogens, including latexbeads opsonised or not with immunoglobulin $\mathrm{G}(\mathrm{IgG})$ or mouse serum (to facilitate entry through the FcR or complement receptors), IgG-opsonized erythrocytes, promastigotes of Leishmania donovani derived from wild-type cells or cell-surface LPG knockout, among other parasites [38]. A mix of ER and endocytic vesicles in the formation of the parasitophorous vacuoles (PVs) during the uptake of different Leishmania spp. was recently shown in macrophages overexpressing ER-tagged green fluorescent protein [41]. The presence of the ER proteins Sec61, Bip/Grp78, and PDI in the phagosome of APCs [38, 41] support the idea that the ER provides the necessary machinery for antigen translocation from the phagosome to the cytoplasm and thus, possibly converges MHC class I and class II antigen cross-presentation [42] (Figure 2). There are several other complementary hypotheses on how peptides cross from phagosomes to cytoplasm during the cross-presentation process $[43,44]$. Neutrophils are short-lived cells (half-life of $4-8 \mathrm{~h}$ in the human circulation) and very active in the phagocytosis of large microbes such as bacteria, parasites, and fungus. Contrary to APCs, neutrophils only contain restricted amounts of ER machinery and are thought to lack the ER-mediated phagocytosis process [38].

Whether ER proteins functionally operate on phagocytosis-mediated infection has not been well characterised yet. An important work has shown that Dictyostelium lacking both ER calreticulin and calnexin present altered phagocytic cup formation and substantial decline in phagocytosis [45]. These two proteins utilise $\mathrm{Ca}$, and their disruption per se affects actin filaments and plasma membrane remodelling during phagocytosis [45]. We recently showed that PDI is critically involved in Leishmania parasite infection in vitro [22]. We showed that phagocytosis of promastigotes (but not amastigotes) of Leishmania chagasi was significantly inhibited by macrophage incubation with the thiol/PDI inhibitors DTNB, bacitracin, phenylarsine oxide, and neutralizing PDI antibody in a parasite redox-dependent way [22]. The phenylarsine response is of particular interest, since this arsenic compound may act similarly to antimonials, widely used in leishmaniasis chemotherapy [46, 47]. PDI preferentially affects parasite internalization and the phagocytosis of the promastigote forms is increased when wild-type PDI is overexpressed in macrophages, an effect opposed by PDI knockdown. At later stages of infection (i.e., after $4 \mathrm{~h}$ ), PDI from promastigote-infected J774 macrophages was immunoprecipitated and subsequently blotted with an antiLeishmania antibody revealing a parasite band at $\sim 94 \mathrm{KDa}$ [16, Figure 10(b), lane 5]. Subsequent removal and analysis of this band by mass fingerprint spectrometry showed a 58\% match with elongation factor 2 (EF2) of L. major (Q4Q259; data not shown). The incubation of purified bovine PDI (Sigma, P3818) and parasites did not yield any detectable protein complexes, suggesting that the macrophage milieu may be important to sustain PDI-EF2 association [22]. Interestingly, Leishmania EF2 has important virulent features and acts as a soluble antigen in lymphocyte stimulation in vitro [48] and in vivo [49]. Moreover, proteomics studies revealed that EF2 is secreted during promastigote differentiation into the amastigote stage with potential immunomodulatory proprieties in animal models [50]. Leishmania EF2 is therefore of particular interest for Leishmania therapeutic interventions such as vaccines. Although our studies did not address the role of the ER in mediating phagocytosis, these data provide compelling evidence for a functional role of ER-PDI in a host-parasite interaction. Other mechanisms underlining PDI-mediated L. chagasi promastigote phagocytosis involves its association to ROS production by phagocyte NADPH oxidase and this is discussed next.

\subsection{PDI and NADPH Oxidase Regulation}

The NADPH oxidase (Nox) family of enzymes uses NADPH as an electron donor to convert oxygen to superoxide anion $\left(\mathrm{O}_{2}{ }^{\bullet-}\right)$, a precursor of $\mathrm{H}_{2} \mathrm{O}_{2}$ and other powerful oxidants such as hydroxyl radical and peroxynitrite (in the presence of nitric oxide), collectively called ROS [3, 51, 52]. Each of the seven oxidase family members is characterized by a distinct catalytic subunit (i.e., Nox1-5 and Duox 1-2), and has differing requirements for additional protein subunits $[51,52]$. The prototypic member of the Nox family, Nox2 oxidase (or gp91 ${ }^{\text {phox }}$ oxidase), is best known for its role in neutrophil and macrophage phagocytosis. Genetic defects in the enzyme are related to chronic granulomatous disease, a condition in which affected children 
suffer from recurrent severe fungal and bacterial infections due to defective phagocyte function [51]. Each Nox isoform forms heterodimers with a lower molecular weight $\mathrm{p} 22^{\text {phox }}$ subunit and is predicted to be membrane-bound. Nox2 is normally quiescent and acutely activated by agonists such as PMA, LPG, and cytokines in a tightly regulated process in which cytosolic subunits (p47 ${ }^{\text {phox }}, \mathrm{p} 67^{\text {phox }}, \mathrm{p} 40^{\text {phox }}$, and Rac1 in the case of macrophages and dendritic cells, or Rac2 in neutrophil) associate with the Nox2-p22 $2^{\text {phox }}$ heterodimer to initiate enzyme activity [51, 52]. Nox2 also has electrogenic features [53] and in APC cells is linked to the regulation of phagosome/lysosome $\mathrm{pH}$ and antigen processing [54, 55]. Usually, phagosome acidity is maintained by a vacuolar ATPase (V-ATPase) that transports protons from the cytosol into the phagosome lumen, therefore regulating the function of lysosome proteases in the fused phagolysosomes. Savina et al. [56-58] have shown that Nox2-derived superoxide in the phagosomal vesicle promptly consumes protons maintaining a higher $\mathrm{pH}$ ambient in dendritic cells during particle internalization, which favours antigen processing and presentation [56-58]. Opposite results were found in macrophages [56-58]. The selective role of Nox 2 in different phagocytic cells remains to be defined. The jury is out on whether the results shown in macrophages (association of Nox2 to Rab27a; a member of Rab family of GTPases) are related to vesicle traffic molecule assembly and quality, or rather associated to degradation processes [59].

Nox complex protein expression and function is greatly affected by redox compounds, and it is especially regulated by PDI with implications for cell signalling [60]. The association of PDI to p22 ${ }^{\text {phox }}$ and other Nox isoforms in different cell types, especially in vascular cells, has been previously described [6062]. A functional and spatial/physical interaction between PDI and the $\mathrm{p} 22^{\text {phox }}$ oxidase subunit was shown in macrophages [22] and more recently between PDI and p47phox in neutrophils [62]. In macrophages, PDI-Nox association was correlated to Leishmania infection in vitro [22]. It is well known that during phagocytosis of Leishmania, Nox2 is activated and parasite uptake is inhibited by antioxidants such as catalase [22]. Intriguingly, in the course of promastigote infection, some parasites evade that stressful condition and convert themselves into intracellular amastigotes, multiplying and resulting in progression to a disease process. Overall, our studies support the view that parasite phagocytosis/infection by macrophages is a redox process mediated by PDI in at least two ways. Initially, PDI-NADPH oxidase increases ROS production generating an oxidizing milieu, which seems to favour promastigote infection. The downstream role of ROS generated by PDI-NADPH oxidase remains unknown but can be related to the unfolded protein response signalling [63] or, similar to PDI-Ero1, to protein folding in the macrophage ER compartment with key implications for antigen processing. Nevertheless, at later stages of infection, macrophage PDI physically associates with Leishmania elongation factor-2 (as discussed earlier).

\subsection{PDI Role on Cell Surface and Pathogen Attachment}

Some viruses envelop their genetic material within a protein-coated capsid in a further lipid membrane layout, for example, influenza virus, baculovirus, hepatitis-C, HIV, and Herpes virus. These enveloped particles require successive steps to successfully entry and infect host cells. They usually first attach onto host receptors (and attachment factors), and their membranes fuse to interact with endosome vesicles that traffic the virus toward the endoplasmic reticulum, where it is uncoated. The proteins are finally transported to the cytosol and nucleus [64] (Figure 2). There is convincing evidence showing that most viral infections are strongly influenced by changes in the redox environment and that host PDI mediates infection of enveloped viruses [65-70].

In the course of HIV infection, the virus first binds to attachment factors, for example, mannose binding C-type lectin receptor and intracellular adhesion molecule (ICAM-3) on the surface of host CD4 ${ }^{+}$ T cells. The glycoprotein 120 (gp120) subunit of the virus envelope binds to immunoglobulin $\mathrm{G}$ of $\mathrm{CD}^{+}$and undergoes conformational changes, allowing the virus to interact with its coreceptors, CXCR4 or CCR5. These interactions favour downstream conversions of gp41 envelope subunit to a competent fusion conformation. Initial studies showed that membrane-impermeable PDI inhibitors and monoclonal antibodies against PDI prevent HIV-1 infection [65]. It was then revealed that the domain D2 of the CD4 has redox-active disulfide bonds and is regulated by thioredoxin [66]. Using membrane-impermeable reducing 
agents (especially arsenical-derived compounds) and labelling thiol reagents, it was demonstrated that $\mathrm{CD}^{+}{ }^{+}$reactive thiols critically drive HIV entry into cells [66]. Work from another group also revealed that PDI, on the surface of HIV-1 target cells, reduces disulfide bonds of the recombinant envelope glycoprotein gp120 (reaction 1, Figure 1), a reaction prevented by the usual PDI inhibitors [67]. Intriguingly, PDI silencing in U373 and HeLa cells had little impact on HIV infection itself as compared to the effect mediated by general thiol inhibitors [68]. The reasons for this discrepancy remain to be elucidated and raise the question whether the reductive effect of PDI is coupled to other redox proteins (e.g., thioredoxin or Nox's) that could amplify virus-CD4 redox association in some cells. It is noteworthy that in these later studies, PDI knockdown on the cell surface was not evident as compared to massive loss of most PDIs within the ER; an observation that supports the idea that PDI in the ER has little impact in HIV-mediated infection [68]. Thiol inhibitors also affect viral fusion as that mediated by the fusion (F) protein from the Paramyxovirus Newcastle disease virus [69]. The overexpression of PDI family members PFDI and ERdj5 has also been shown to significantly catalyze the reduction of thiols in F protein, facilitating membrane fusion [70]. There is evidence suggesting a possible association between PDI and infection mediated by some members of the of Herpesviridae viruses family [71].

PDI is also implicated in the attachment of some bacteria from different species of Chlamydia [7274]. Chlamydia is an obligatory intracellular pathogen that causes diverse diseases in humans. The most common species are Chlamydia trachomatis, which is sexually transmitted and can cause blindness and infertility, and $C$. pneumoniae, which affects the respiratory tract. CHO cells have impaired endogenous PDI expression due to a defect in truncated mRNA processing, thus providing a valuable model to understand the effect of PDI-mediated cell-cell interaction and infection. These cells are very resistant to Chlamydia infection showing impaired attachment, an effect restored by ectopic expression of PDI [73]. Similar to HIV infection, the molecular mechanisms most likely include the reductive activity of PDI (reaction 1, Figure 1) on the surface of $\mathrm{CHO}$ cells [72].

\subsection{PDI and Antigen Translocation from ER to Cytosol}

Crossing the endoplasmic reticulum (ER) membrane is an irreversible process for most proteins. In some cases, however, this flow is reversed and misfolded proteins retained in the ER are retrotranslocated to the cytosol via ERAD to be degraded by the proteasome. This pathway is also exploited by small pathogens, especially non-enveloped viruses and some bacterial toxins, to gain access to the cytosol. In these cases, antigenic particles that reach the ER by different means suffer molecular redox rearrangements and binding to PDI allowing them to be transported back to the cytosol or nucleus. Well-known examples are infections mediated by Polyomaviruses (Py) and Simian virus 40 (SV40) extensively studied in the field of carcinogenesis. After SV40 interaction with the GM1 receptor on the cell surface, the particle enters the host cell through endocytosis and traffics via the caveosome (a particular caveolin containing endosome with neutral $\mathrm{pH}$ ) towards the ER compartment $[75,76]$. SV40-coated pentamers are linked to each other by disulfide bonds between cysteine 104 (C104). Further isomerisation in the ER is crucial for the viral uncoating process. In vitro cell screening shows that among all ER-resident proteins, PDI and ERp57 more specifically regulate SV40 infection [75]. PDI silencing substantially decreases SV40 infection that is also dependent on some redox sensitive cysteines on the viral particle [75]. PDI cooperation with ERassociated ERAD proteins Derlin-1 and Sel1L is Ca dependent and facilitates SV40 traffic through ERAD [75]. A similar pathway is used by some nonobligatory intracellular bacteria that exert their effect through production of potent endotoxins, such as diphtheria toxin (DT) and cholera toxin (CT). These proteins function similarly to some plant toxins, such as ricin and abrin. Conversion into toxic proteins involves cleavage of their interchain disulfide bond, allowing them to traffic into the endocytic pathway within the host cell $[77,78]$. In humans, CT is derived from the Bacterium Vibrio cholerae that causes cholera disease and has 2 subunits (A1 and A2). The protein first attaches to the host cell surface via GM1 and the subunit A2, which contains a KDEL sequence, and is transported back to the ER (see earlier discussion). There, PDI reduces and unfolds A2 and A1 that exit the ER via the Sec61 channel into the cytosol. PDI in the 
reduced state (reaction 1, Figure 1) binds to the toxin and subsequent oxidation of PDI, probably via Ero1 $\alpha$, enables the release of $\mathrm{CT}$ toxin $[79,80]$. The active polypeptide A1 efficiently modifies a heterotrimeric $\mathrm{G}$ protein in the cytosol that leads to massive loss of chlorine and water secretion by intestinal epithelial cells in mammals, resulting in severe diarrhoea.

\section{CONCLUSION}

In this article we have reviewed the main cellular aspects of PDI-mediated host pathogen interactions and the pathways that are involved in viral, bacterial (including bacterial toxins), and parasitic infections. A number of cellular mechanisms through which PDI modulates some specific cellular pathways in immune cells have been described, such as redox-sensitive attachment, antigen presentation in the ER and exit from it, and association to phagosome and ROS production by NADPH oxidase (Figure 2). Many of these responses are antigen-specific and the precise mechanisms of action remain to be fully elucidated, especially in the context of redox changes in cross-presentation phenomena. Moreover, little is known about the role of PDI in infection per se, as well as how PDI signals to a more integrated cellular response to stress [63]. PDI global knockout mice are only viable until birth, but partial gene-modified mice and also modified pathogens will help to reveal the significant redox role of PDI and its redox partners. Overall, PDI is a key regulator that may propagate or limit the severity of the infection processes, depending on the infectious organism involved. A better understanding of these complex regulatory steps will provide insightful information on the redox role and coevolutional biological process, and assist the development of more specific therapeutic strategies in pathogen-mediated infections.

\section{ABBREVIATIONS}

ER: $\quad$ Endoplasmic reticulum

MHC: Major histocompatibility complex

$\mathrm{H}_{2} \mathrm{O}_{2}$ : Hydrogen peroxide

ERp57: A member of PDI family also know as glucose-regulated protein or 58-kD (GRP58)

NF-kB: Factor nuclear kappa B

AP-1: Activator protein 1

STAT-3: Signal transducer and activator of transcription 3.

\section{ACKNOWLEDGMENTS}

The authors thank Daniel C. Pimenta for mass spectrometry experiments (Centre for Applied Toxinology CAT/CEPID, Butantan Institute). They also thank Dr. João Wosniak Jr. from Vascular Biology Laboratory, Heart Institute (InCor) for helpful discussion. This paper was supported by Auxílio Fundação de Amparo à Pesquisa do Estdado de São Paulo (FAPESP) and Conselho Nacional de Pesquisa (CNPq), Instituto do Milênio Redoxoma; Fundação EJ Zerbini (InCor). A. M. Shah, S. Ioannis and C. X. C. Santos are also supported by the British Heart Foundation and a Leducq Fondation Transatlantic Network of Excellence award.

\section{REFERENCES}

[1] E. Fenouillet, R. Barbouche, and I. M. Jones, "Cell entry by enveloped viruses: redox considerations for HIV and SARS-coronavirus," Antioxidants \& Redox Signaling, vol. 9, no. 8, pp. 1009-1034, 2007.

[2] C. F. Nathan and J. B. Hibbs, "Role of nitric oxide synthesis in macrophage antimicrobial activity," Current Opinion in Immunology, vol. 3, no. 1, pp. 65-70, 1991.

[3] O. Augusto, M. G. Bonini, A. M. Amanso, E. Linares, C. C. X. Santos, and S. L. De Menezes, "Nitrogen dioxide and carbonate radical anion: two emerging radicals in biology," Free Radical Biology and Medicine, vol. 32, no. 9, pp. 841-859, 2002. 
[4] C. Appenzeller-Herzog and L. Ellgaard, "The human PDI family: versatility packed into a single fold," Biochimica et Biophysica Acta, vol. 1783, no. 4, pp. 535-548, 2008.

[5] F. Hatahet and L. W. Ruddock, "Protein disulfide isomerase: a critical evaluation of its function in disulfide bond formation," Antioxidants \& Redox Signaling, vol. 11, no. 11, pp. 2807-2850, 2009.

[6] R. Noiva, "Protein disulfide isomerase: the multifunctional redox chaperone of the endoplasmic reticulum," Seminars in Cell and Developmental Biology, vol. 10, no. 5, pp. 481-493, 1999.

[7] E. Gross, C. S. Sevier, N. Heldman et al., "Generating disulfides enzymatically: reaction products and electron acceptors of the endoplasmic reticulum thiol oxidase Ero1p," Proceedings of the National Academy of Sciences of the United States of America, vol. 103, no. 2, pp. 299-304, 2006.

[8] B. P. Tu and J. S. Weissman, "The FAD- and $\mathrm{O}_{2}$-dependent reaction cycle of Ero1-mediated oxidative protein folding in the endoplasmic reticulum," Molecular Cell, vol. 10, no. 5, pp. 983-994, 2002.

[9] R. Xiao, B. Wilkinson, A. Solovyov et al., "The contributions of protein bisulfide isomerase and its homologues to oxidative protein folding in the yeast endoplasmic reticulum," Journal of Biological Chemistry, vol. 279, no. 48, pp. 49780-49786, 2004.

[10] B. Wilkinson and H. F. Gilbert, "Protein disulfide isomerase," Biochimica et Biophysica Acta, vol. 1699, no. 1-2, pp. 35-44, 2004.

[11] T. J. Tavender, J. J. Springate, and N. J. Bulleid, "Recycling of peroxiredoxin IV provides a novel pathway for disulphide formation in the endoplasmic reticulum," The EMBO Journal, vol. 29, no. 24, pp. 4185-4197, 2010.

[12] E. Zito, E. P. Melo, Y. Yang, Å. Wahlander, T. A. Neubert, and D. Ron, "Oxidative protein folding by an endoplasmic reticulum-localized peroxiredoxin," Molecular Cell, vol. 40, no. 5, pp. 787-797, 2010.

[13] E. Margittai and G. Bánhegyi, "Oxidative folding in the endoplasmic reticulum: towards a multiple oxidant hypothesis?" FEBS Letters, vol. 584, no. 14, pp. 2995-2998, 2010.

[14] K. Terada, P. Manchikalapudi, R. Noiva, H. O. Jauregui, R. J. Stockert, and M. L. Schilsky, "Secretion, surface localization, turnover, and steady state expression of protein disulfide isomerase in rat hepatocytes," Journal of Biological Chemistry, vol. 270, no. 35, pp. 20410-20416, 1995.

[15] C. Turano, S. Coppari, F. Altieri, and A. Ferraro, "Proteins of the PDI family: unpredicted non-ER locations and functions," Journal of Cellular Physiology, vol. 193, no. 2, pp. 154-163, 2002.

[16] Y. Ben Achour, M. Chenik, H. Louzir, and K. Dellagi, "Identification of a disulfide isomerase protein of Leishmania major as a putative virulence factor," Infection and Immunity, vol. 70, no. 7, pp. 3576-3585, 2002.

[17] S. N. Molteni, A. Fassio, M. R. Ciriolo et al., "Glutathione limits Ero1-dependent oxidation in the endoplasmic reticulum," Journal of Biological Chemistry, vol. 279, no. 31, pp. 32667-32673, 2004.

[18] K. S. Na, B. C. Park, M. Jang et al., "Protein disulfide isomerase is cleaved by caspase-3 and -7 during apoptosis," Molecules and Cells, vol. 24, no. 2, pp. 261-267, 2007.

[19] M. P. Posada-Vergara, J. A. L. Lindoso, J. E. Tolezano, V. L. Pereira-Chioccola, M. V. Silva, and H. Goto, "Tegumentary leishmaniasis as a manifestation of immune reconstitution inflammatory syndrome in 2 patients with AIDS," Journal of Infectious Diseases, vol. 192, no. 10, pp. 1819-1822, 2005.

[20] M. J. McConville and E. Handman, "The molecular basis of Leishmania pathogenesis," International Journal for Parasitology, vol. 37, no. 10, pp. 1047-1051, 2007.

[21] J. J. Shaw, "Further thoughts on the use of the name Leishmania (Leishmania) infantum chagasi for the aetiological agent of American visceral leishmaniasis," Memorias do Instituto Oswaldo Cruz, vol. 101, no. 5, pp. 577-579, 2006.

[22] C. X. C. Santos, B. S. Stolf, P. V. A. Takemoto et al., "Protein disulfide isomerase (PDI) associates with NADPH oxidase and is required for phagocytosis of Leishmania chagasi promastigotes by macrophages," Journal of Leukocyte Biology, vol. 86, no. 4, pp. 989-998, 2009.

[23] B. X. Hong and L. Soong, "Identification and enzymatic activities of four protein disulfide isomerase (PDI) isoforms of Leishmania amazonensis," Parasitology Research, vol. 102, no. 3, pp. 437-446, 2008.

[24] F. Benhnini, M. Chenik, D. Laouini, H. Louzir, P. A. Cazenave, and K. Dellagi, "Comparative evaluation of two vaccine candidates against experimental leishmaniasis due to Leishmania major infection in four inbred mouse strains," Clinical and Vaccine Immunology, vol. 16, no. 11, pp. 1529-1537, 2009.

[25] M. P. Hsu, M. L. Munich, and J. C. Boothroyd, "A developmentally regulated gene of trypanosomes encodes a homologue of rat protein-disulfide isomerase and phosphoinositol-phospholipase C," Biochemistry, vol. 28, no. 15, pp. 6440-6446, 1989. 
[26] J. Rubotham, K. Woods, J. A. Garcia-Salcedo, E. Pays, and D. P. Nolan, "Characterization of two protein disulfide isomerases from the endocytic pathway of bloodstream forms of Trypanosoma brucei," Journal of Biological Chemistry, vol. 280, no. 11, pp. 10410-10418, 2005.

[27] I. Florenta, E. Mouray, F. Dali Ali et al., "Cloning of Plasmodium falciparum protein disulfide isomerase homologue by affinity purification using the antiplasmodial inhibitor 1,4-bis $\{3$-[N-(cyclohexyl methyl)amino]propyl\}piperazine," FEBS Letters, vol. 484, no. 3, pp. 246-252, 2000.

[28] B. Mahajan, R. Noiva, A. Yadava et al., "Protein disulfide isomerase assisted protein folding in malaria parasites," International Journal for Parasitology, vol. 36, no. 9, pp. 1037-1048, 2006.

[29] B. Meek, J. W. Back, V. N. A. Klaren, D. Speijer, and R. Peek, "Protein disulfide isomerase of Toxoplasma gondii is targeted by mucosal IgA antibodies in humans," FEBS Letters, vol. 522, no. 1-3, pp. 104-108, 2002.

[30] R. E. Mares, P. D. Magaña, S. G. Meléndez-López, A. F. Licea, J. M. Cornejo-Bravo, and M. A. Ramos, "Oxidative folding and reductive activities of EhPDI, a protein disulfide isomerase from Entamoeba histolytica," Parasitology International, vol. 58, no. 3, pp. 311-313, 2009.

[31] S. Bertholet, R. Goldszmid, A. Morrot et al., "Leishmania antigens are presented to CD8 ${ }^{+} \mathrm{T}$ cells by a transporter associated with antigen processing-independent pathway in vitro and in vivo," Journal of Immunology, vol. 177, no. 6, pp. 3525-3533, 2006.

[32] B. Park, S. Lee, E. Kim et al., "Redox regulation facilitates optimal peptide selection by MHC class I during antigen processing," Cell, vol. 127, no. 2, pp. 369-382, 2006.

[33] Y. Kim, K. Kang, I. Kim et al., "Molecular mechanisms of MHC class I-antigen processing: redox considerations," Antioxidants \& Redox Signaling, vol. 11, no. 4, pp. 907-936, 2009.

[34] S. Lee, B. Park, K. Kang, and K. Ahn, "Redox-regulated export of the major histocompatibility complex class I-peptide complexes from the endoplasmic reticulum," Molecular Biology of the Cell, vol. 20, no. 14, pp. 32853294, 2009.

[35] K. Kang, B. Park, C. Oh, K. Cho, and K. Ahn, "A role for protein disulfide isomerase in the early folding and assembly of MHC class I molecules," Antioxidants \& Redox Signaling, vol. 11, no. 10, pp. 2553-2561, 2009.

[36] R. Abele and R. Tampé, "Peptide trafficking and translocation across membranes in cellular signaling and selfdefense strategies," Current Opinion in Cell Biology, vol. 21, no. 4, pp. 508-515, 2009.

[37] M. Rabinovitch, "Professional and non-professional phagocytes: an introduction," Trends in Cell Biology, vol. 5, no. 3, pp. 85-87, 1995.

[38] J. Garin, R. Diez, S. Kieffer et al., "The phagosome proteome: insight into phagosome functions," Journal of Cell Biology, vol. 152, no. 1, pp. 165-180, 2001.

[39] M. Desjardins, "ER-mediated phagocytosis: a new membrane for new functions," Nature Reviews Immunology, vol. 3, no. 4, pp. 280-291, 2003.

[40] E. Gagnon, S. Duclos, C. Rondeau et al., "Endoplasmic reticulum-mediated phagocytosis is a mechanism of entry into macrophages," Cell, vol. 110, no. 1, pp. 119-131, 2002.

[41] B. Ndjamen, B. H. Kang, K. Hatsuzawa, and P. E. Kima, "Leishmania parasitophorous vacuoles interact continuously with the host cell's endoplasmic reticulum; parasitophorous vacuoles are hybrid compartments," Cellular Microbiology, vol. 12, no. 10, pp. 1480-1494, 2010.

[42] M. Houde, S. Bertholet, E. Gagnon et al., "Phagosomes are competent organelles for antigen cross-presentation," Nature, vol. 425, no. 6956, pp. 402-406, 2003.

[43] J. M. Vyas, A. G. van der Veen, and H. L. Ploegh, "The known unknowns of antigen processing and presentation," Nature Reviews Immunology, vol. 8, no. 8, pp. 607-618, 2008.

[44] N. Touret, P. Paroutis, M. Terebiznik et al., "Quantitative and dynamic assessment of the contribution of the ER to phagosome formation," Cell, vol. 123, no. 1, pp. 157-170, 2005.

[45] A. Müller-Taubenberger, A. N. Lupas, H. Li, M. Ecke, E. Simmeth, and G. Gerisch, "Calreticulin and calnexin in the endoplasmic reticulum are important for phagocytosis," The EMBO Journal, vol. 20, no. 23, pp. 6772-6782, 2001.

[46] Ashutosh, S. Sundar, and N. Goyal, "Molecular mechanisms of antimony resistance in Leishmania," Journal of Medical Microbiology, vol. 56, no. 2, pp. 143-153, 2007.

[47] S. Wyllie, M. L. Cunningham, and A. H. Fairlamb, "Dual action of antimonial drugs on thiol redox metabolism in the human pathogen Leishmania donovani," Journal of Biological Chemistry, vol. 279, no. 38, pp. 39925-39932, 2004. 
[48] S. K. Gupta, B. S. Sisodia, S. Sinha et al., "Proteomic approach for identification and characterization of novel immunostimulatory proteins from soluble antigens of Leishmania donovani promastigotes," Proteomics, vol. 7 , no. 5, pp. 816-823, 2007.

[49] P. Probst, E. Stromberg, H. W. Ghalib et al., "Identification and characterization of T cell-stimulating antigens from Leishmania by CD4 T cell expression cloning," Journal of Immunology, vol. 166, no. 1, pp. 498-505, 2001.

[50] M. Chenik, S. Lakhal, N. Ben Khalef, L. Zribi, H. Louzir, and K. Dellagi, "Approaches for the identification of potential excreted/secreted proteins of Leishmania major parasites," Parasitology, vol. 132, no. 4, pp. 493-509, 2006.

[51] A. van der Vliet, "NADPH oxidases in lung biology and pathology: host defense enzymes, and more," Free Radical Biology and Medicine, vol. 44, no. 6, pp. 938-955, 2008.

[52] J. D. Lambeth, "NOX enzymes and the biology of reactive oxygen," Nature Reviews Immunology, vol. 4, no. 3, pp. 181-189, 2004.

[53] L. M. Henderson, J. B. Chappell, and O. T. G. Jones, “The superoxide-generating NADPH oxidase of human neutrophils is electrogenic and associated with an $\mathrm{H}^{+}$channel," Biochemical Journal, vol. 246, no. 2, pp. 325329, 1987.

[54] A. R. Cross and A. W. Segal, "The NADPH oxidase of professional phagocytes—prototype of the NOX electron transport chain systems," Biochimica et Biophysica Acta, vol. 1657, no. 1, pp. 1-22, 2004.

[55] A. Savina and S. Amigorena, "Phagocytosis and antigen presentation in dendritic cells," Immunological Reviews, vol. 219, no. 1, pp. 143-156, 2007.

[56] A. Savina, C. Jancic, S. Hugues et al., "NOX2 controls phagosomal pH to regulate antigen processing during crosspresentation by dendritic cells," Cell, vol. 126, no. 1, pp. 205-218, 2006.

[57] C. Jancic, A. Savina, C. Wasmeier et al., "Rab27a regulates phagosomal pH and NADPH oxidase recruitment to dendritic cell phagosomes," Nature Cell Biology, vol. 9, no. 4, pp. 367-378, 2007.

[58] A. R. Mantegazza, A. Savina, M. Vermeulen et al., "NADPH oxidase controls phagosomal pH and antigen crosspresentation in human dendritic cells," Blood, vol. 112, no. 12, pp. 4712-4722, 2008.

[59] J. Huang, V. Canadien, G. Y. Lam et al., "Activation of antibacterial autophagy by NADPH oxidases," Proceedings of the National Academy of Sciences of the United States of America, vol. 106, no. 15, pp. 62266231, 2009.

[60] F. R. M. Laurindo, D. C. Fernandes, A. M. Amanso, L. R. Lopes, and C. X. C. Santos, "Novel role of protein disulfide isomerase in the regulation of NADPH oxidase activity: pathophysiological implications in vascular diseases," Antioxidants \& Redox Signaling, vol. 10, no. 6, pp. 1101-1113, 2008.

[61] M. Janiszewski, L. R. Lopes, A. O. Carmo et al., "Regulation of NAD(P)H oxidase by associated protein disulfide isomerase in vascular smooth muscle cells," Journal of Biological Chemistry, vol. 280, no. 49, pp. 40813-40819, 2005.

[62] A. M. de A. Paes, S. Veríssimo-Filho, L. L. Guimarães et al., "Protein disulfide isomerase redox-dependent association with p47phox: evidence for an organizer role in leukocyte NADPH oxidase activation," Journal of Leukocyte Biology, vol. 90, no. 4, pp. 799-810, 2011.

[63] C. X. Santos, L. Y. Tanaka, J. Wosniak, and F. R. Laurindo, "Mechanisms and implications of reactive oxygen species generation during the unfolded protein response: roles of endoplasmic reticulum oxidoreductases, mitochondrial electron transport, and NADPH oxidase," Antioxidants \& Redox Signaling, vol. 11, no. 10, pp. 2409-2427, 2009.

[64] A. E. Smith and A. Helenius, "How viruses enter animal cells," Science, vol. 304, no. 5668, pp. 237-242, 2004.

[65] H. J. P. Ryser, E. M. Levy, R. Mandel, and G. J. DiSciullo, "Inhibition of human immunodeficiency virus infection by agents that interfere with thiol-disulfide interchange upon virus-receptor interaction," Proceedings of the National Academy of Sciences of the United States of America, vol. 91, no. 10, pp. 4559-4563, 1994.

[66] L. J. Matthias, P. T. W. Yam, X. M. Jiang et al., "Disulfide exchange in domain 2 of CD4 is required for entry of HIV-I," Nature Immunology, vol. 3, no. 8, pp. 727-732, 2002.

[67] A. Gallina, T. M. Hanley, R. Mandel et al., "Inhibitors of protein-disulfide isomerase prevent cleavage of disulfide bonds in receptor-bound glycoprotein 120 and prevent HIV-1 entry," Journal of Biological Chemistry, vol. 277, no. 52, pp. 50579-50588, 2002.

[68] W. Ou and J. Silver, "Role of protein disulfide isomerase and other thiol-reactive proteins in HIV-1 envelope protein-mediated fusion," Virology, vol. 350, no. 2, pp. 406-417, 2006. 
[69] S. Jain, L. W. McGinnes, and T. G. Morrison, "Thiol/disulfide exchange is required for membrane fusion directed by the Newcastle disease virus fusion protein," Journal of Virology, vol. 81, no. 5, pp. 2328-2339, 2007.

[70] S. Jain, L. W. McGinnes, and T. G. Morrison, "Overexpression of thiol/disulfide isomerases enhances membrane fusion directed by the newcastle disease virus fusion protein," Journal of Virology, vol. 82, no. 24, pp. 1203912048, 2008.

[71] H. O. Al-Barazi and A. M. Colberg-Poley, "The human cytomegalovirus UL37 immediate-early regulatory protein is an integral membrane $\mathrm{N}$-glycoprotein which traffics through the endoplasmic reticulum and Golgi apparatus," Journal of Virology, vol. 70, no. 10, pp. 7198-7208, 1996.

[72] S. Abromaitis and R. S. Stephens, "Attachment and entry of chlamydia have distinct requirements for host protein disulfide isomerase," PLoS Pathogens, vol. 5, no. 4, Article ID e1000357, 2009.

[73] C. G. Conant and R. S. Stephens, "Chlamydia attachment to mammalian cells requires protein disulfide isomerase," Cellular Microbiology, vol. 9, no. 1, pp. 222-232, 2007.

[74] C. H. Davis, J. E. Raulston, and P. B. Wyrick, "Protein disulfide isomerase, a component of the estrogen receptor complex, is associated with Chlamydia trachomatis serovar E attached to human endometrial epithelial cells," Infection and Immunity, vol. 70, no. 7, pp. 3413-3418, 2002.

[75] M. Schelhaas, J. Malmström, L. Pelkmans et al., "Simian virus 40 depends on ER protein folding and quality control factors for entry into host cells," Cell, vol. 131, no. 3, pp. 516-529, 2007.

[76] J. Gilbert, W. Ou, J. Silver, and T. Benjamin, "Downregulation of protein disulfide isomerase inhibits infection by the mouse polyomavirus," Journal of Virology, vol. 80, no. 21, pp. 10868-10870, 2006.

[77] E. P. Feener, W. C. Shen, and H. J. P. Ryser, "Cleavage of disulfide bonds in endocytosed macromolecules. A processing not associated with lysosomes or endosomes," Journal of Biological Chemistry, vol. 265, no. 31, pp. 18780-18785, 1990.

[78] H. J. P. Ryser, R. Mandel, and F. Ghani, "Cell surface sulfhydryls are required for the cytotoxicity of diphtheria toxin but not of ricin in Chinese hamster ovary cells," Journal of Biological Chemistry, vol. 266, no. 28, pp. 18439-18442, 1991.

[79] B. Tsai and T. A. Rapoport, "Unfolded cholera toxin is transferred to the ER membrane and released from protein disulfide isomerase upon oxidation by Ero1," Journal of Cell Biology, vol. 159, no. 2, pp. 207-215, 2002.

[80] P. Moore, K. M. Bernardi, and B. Tsai, "The erol $\alpha$-PDI redox cycle regulates retro-translocation of cholera toxin," Molecular Biology of the Cell, vol. 21, no. 7, pp. 1305-1313, 2010.

\section{This article should be cited as follows:}

Beatriz S. Stolf, Ioannis Smyrnias, Lucia R. Lopes, Alcione Vendramin, Hiro Goto, Francisco R. M. Laurindo, Ajay M. Shah, and Celio X. C. Santos, "Protein Disulfide Isomerase and Host-Pathogen Interaction,” TheScientificWorldJOURNAL, vol. 11, pp. 1749-1761, 2011. 

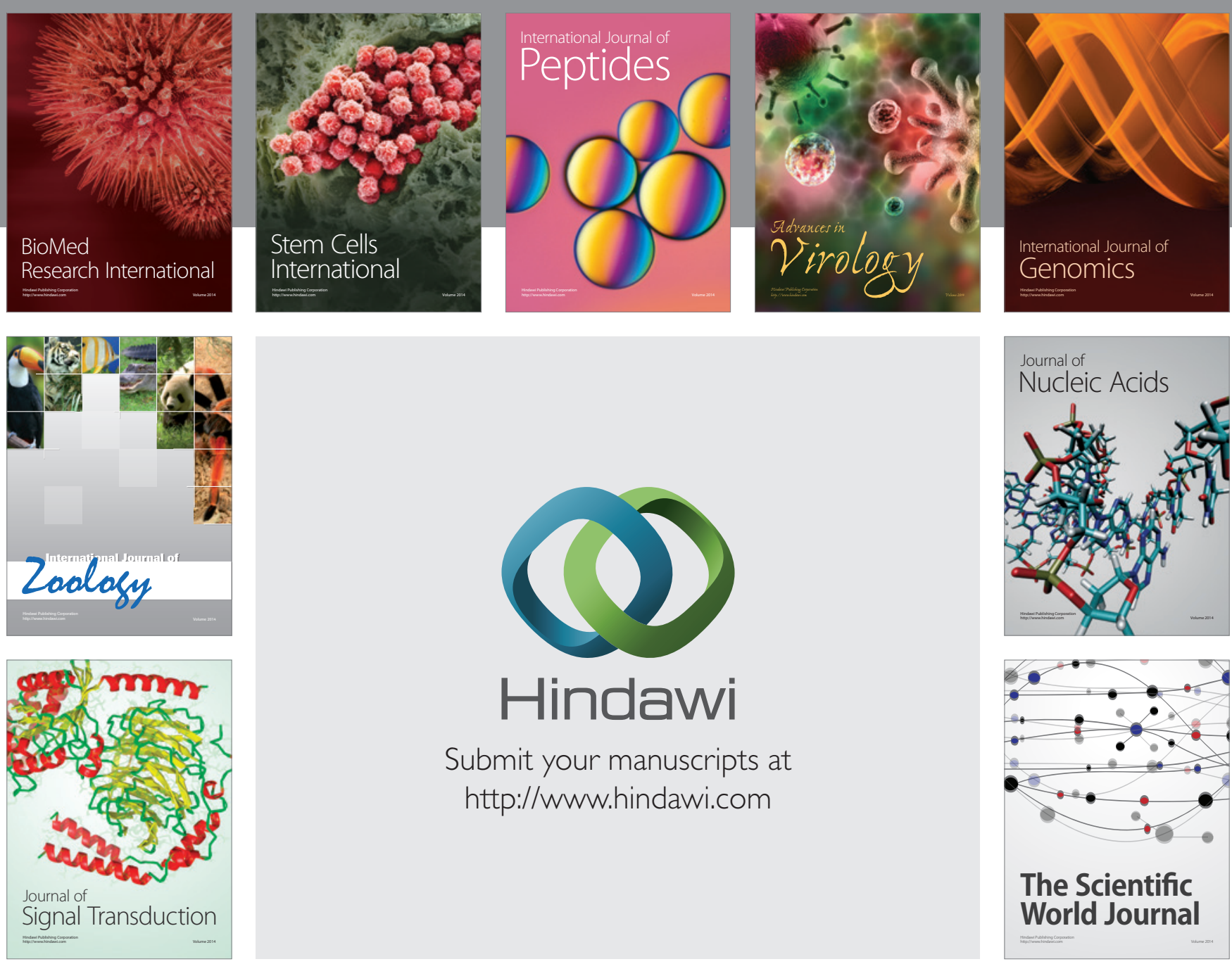

Submit your manuscripts at

http://www.hindawi.com
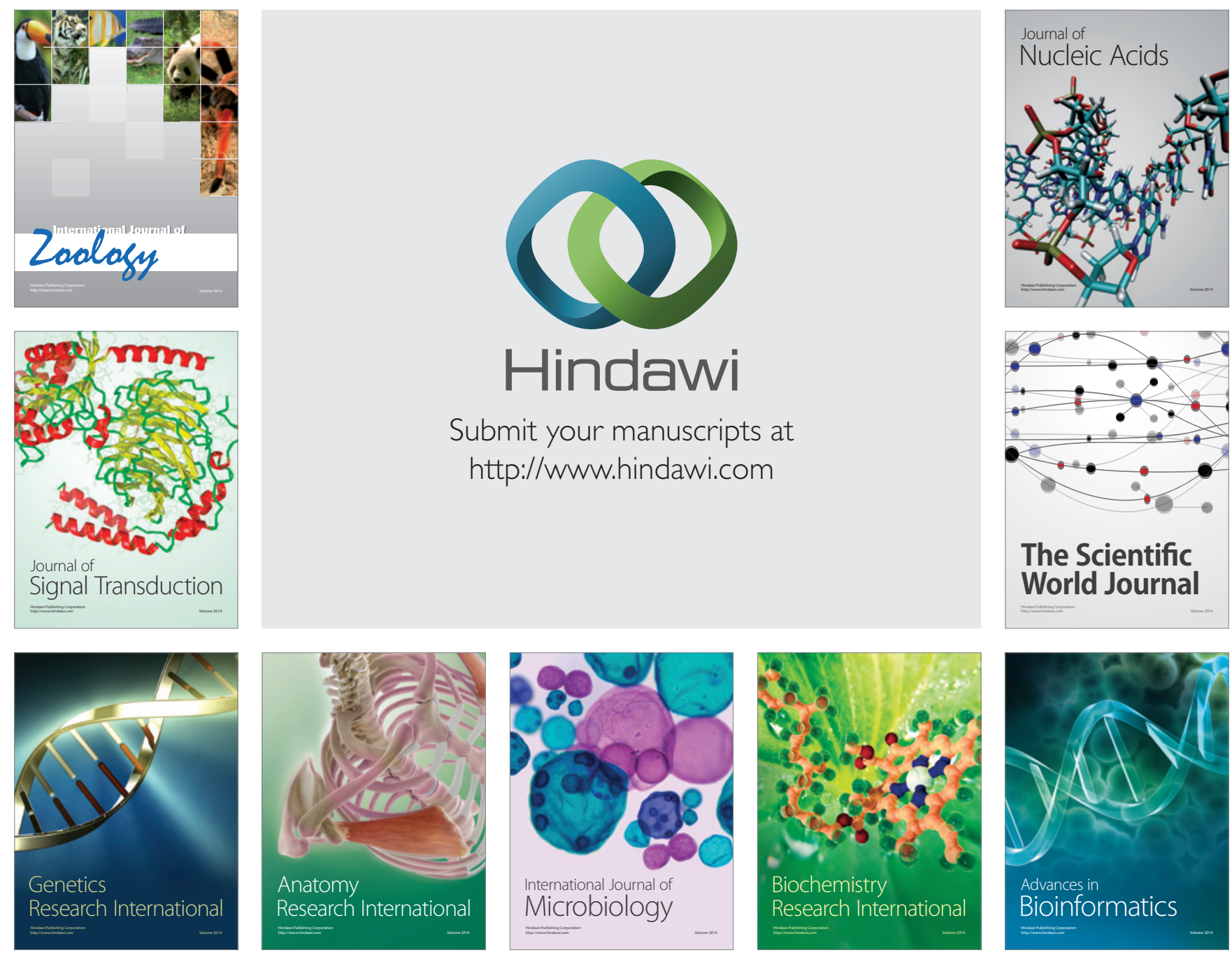

The Scientific World Journal
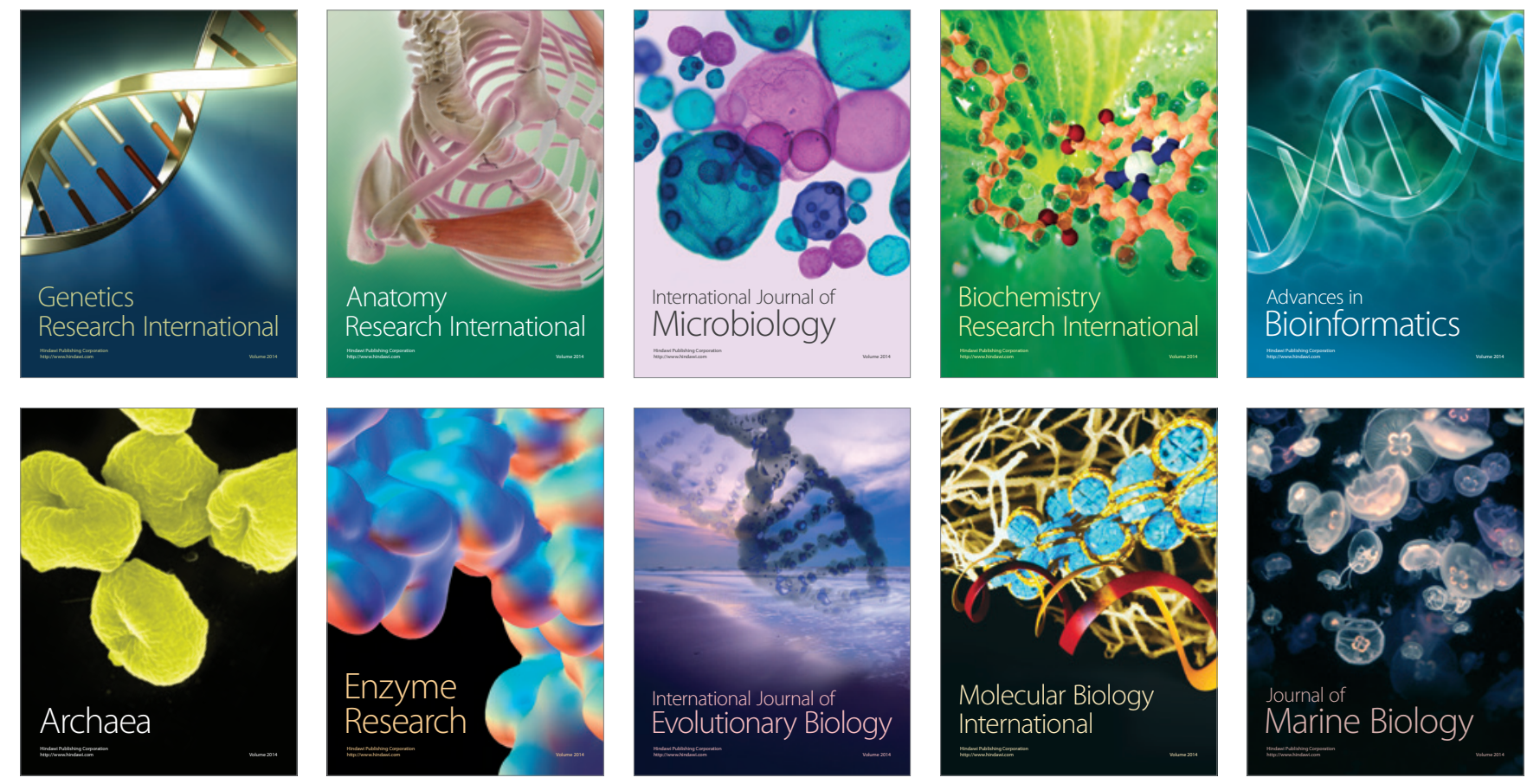